\title{
Biocache-store: Command-Line Interface tool for managing occurrence records
}

\author{
Manash Shah ${ }^{\ddagger}$, Markus Skyttner ${ }^{\ddagger}$ \\ ‡ Swedish Museum of Natural History, Stockholm, Sweden
}

Corresponding author: Manash Shah (manash.shah@nrm.se)

Received: 14 Jul 2017 | Published: 25 Jul 2017

Citation: Shah M, Skyttner M (2017) Biocache-store: Command-Line Interface tool for managing occurrence records. Proceedings of TDWG 1: e15071. https://doi.org/10.3897/tdwgproceedings.1.15071

\begin{abstract}
The Atlas of Living Australia (ALA) (https://www.ala.org.au/) is a collaborative, open infrastructure for sharing biodiversity data. The software and tools are publicly available for reuse and customization (https://github.com/AtlasOfLivingAustralia). GBIF Sweden, hosted at the Swedish Museum of Natural History, is in the process of deploying the Swedish data portal (BioAtlas Sweden - https://bioatlas.se/) adopting the components from ALA. GBIF Sweden serves over 66 million occurrence records and is one of the biggest contributors to the GBIF Network. The requirement for managing of this volume of data led to implementation of the components from ALA in the Swedish data portal.
\end{abstract}

This presentation provides a technical overview of one of the tools from ALA, the Biocachestore. The functionalities for loading, sampling, processing and indexing of occurrence records using the command-line interface tool will be covered in depth. Additional functionalities to support outliers detection, duplicate detection and identifying extra-limital outliers based on authoritative distribution polygons for taxa shall be demonstrated. Following the demonstration, there will be time to explore the features of the presented tools.

\section{Keywords}

Biocache-store, Atlas of Living Australia 


\section{Presenting author}

Manash Shah 